TRANSACTIONS OF THE

AMERICAN MATHEMATICAL SOCIETY

Volume 365, Number 4, April 2013, Pages 2223-2240

S 0002-9947(2012)05712-6

Article electronically published on September 19, 2012

\title{
FIELDS OF ALGEBRAIC NUMBERS WITH BOUNDED LOCAL DEGREES AND THEIR PROPERTIES
}

\begin{abstract}
SARA CHECCOLI
Abstract. We provide a characterization of infinite algebraic Galois extensions of the rationals with uniformly bounded local degrees, giving a detailed proof of all the results in Checcoli and Zannier's paper (2011) and obtaining relevant generalizations for them. In particular we show that for an infinite Galois extension of the rationals the following three properties are equivalent: having uniformly bounded local degrees at every prime; having uniformly bounded local degrees at all but finitely many primes; having Galois group of finite exponent. The proof of this result enlightens interesting connections with Zelmanov's work on the Restricted Burnside Problem. We give a formula to explicitly compute bounds for the local degrees of an infinite extension in some special cases. We relate the uniform boundedness of the local degrees to other properties: being a subfield of $\mathbb{Q}^{(d)}$, which is defined as the compositum of all number fields of degree at most $d$ over $\mathbb{Q}$; being generated by elements of uniformly bounded degree. We prove that the above properties are equivalent for abelian extensions, but not in general; we provide counterexamples based on group-theoretical constructions with extraspecial groups and their modules, for which we give explicit realizations.
\end{abstract}

\section{INTRODUCTION}

This work is a study of the relations between some properties which can occur for an infinite algebraic extension $K$ of the rationals.

The first property we shall consider is the uniform boundedness of the local degrees of $K$, namely the existence of a constant $b$, depending only on the field $K$, such that for every prime number $p$ and every place $v_{p}$ of $K$ which extends the $p$-adic one, the completion of $K$ with respect to $v_{p}$ is a finite extension of $\mathbb{Q}_{p}$ of degree bounded by $b$.

The second property is the inclusion of the extension $K$ in a field $\mathbb{Q}^{(d)}$, which is defined as the compositum in the algebraic closure of the rationals $\mathbb{Q}^{\text {alg }}$ of all number fields of degree at most $d$ over $\mathbb{Q}$.

The inquiry into the relation between these two properties was motivated by Bombieri and Zannier's paper [1], which studies the Northcott property (of the finiteness of elements of bounded absolute Weil height) for certain infinite extensions of $\mathbb{Q}$. The paper considers in particular the field $\mathbb{Q}^{(d)}$, and the Northcott property is proved for the compositum of all abelian extensions of $\mathbb{Q}$ of uniformly bounded degree, while for $\mathbb{Q}^{(d)}$ the question remains open. In this proof a crucial role is played by the uniform boundedness of the local degrees of the field $\mathbb{Q}^{(d)}$.

Received by the editors December 21, 2010 and, in revised form, March 14, 2011, March 29, 2011, April 7, 2011, July 15, 2011, August 31, 2011 and September 18, 2011.

2010 Mathematics Subject Classification. Primary 11S15, $11 \mathrm{R} 32$.

(C) 2012 American Mathematical Society Reverts to public domain 28 years from publication 
The considerations made in [1] led to the question, not free of independent interest, of whether every infinite algebraic extension of $\mathbb{Q}$ with uniformly bounded local degrees is contained in $\mathbb{Q}^{(d)}$ for some positive integer $d$. This question was negatively answered in [2], Theorem 1.1, where the authors provide a counterexample built up from a certain family of $p q$-groups provided by A. Lucchini. In this work we ask, more generally, if for an algebraic extension of $\mathbb{Q}$, there are properties which are equivalent to having uniformly bounded local degrees.

We also ask whether the uniform boundedness of the local degrees at every prime is equivalent to the uniform boundedness of the local degrees at almost every prime, that is, at all but a finite number of primes.

The study of the structure of $\mathbb{Q}^{(d)}$ revealed some unexpected sides. These arise when we consider a third property for an infinite algebraic extension $K$ of $\mathbb{Q}$, that is, whether every number field contained in $K$ can be generated by elements of degree bounded by a constant depending only on $K$. A field with this property is certainly contained in $\mathbb{Q}^{(d)}$ for some positive integer $d$ and one could ask whether this condition is equivalent to being a subfield of $\mathbb{Q}^{(d)}$.

In this work we give a complete answer to all these questions, proving the following result.

Theorem 1. Let $K / \mathbb{Q}$ be an infinite Galois extension. Then the following conditions are equivalent:

(1) $K$ has uniformly bounded local degrees at every prime;

(2) K has uniformly bounded local degrees at almost every prime;

(3) $\operatorname{Gal}(K / \mathbb{Q})$ has finite exponent.

Moreover, if $K / \mathbb{Q}$ is abelian, then the three properties:

(a) $K$ has uniformly bounded local degrees;

(b) $K$ is contained in $\mathbb{Q}^{(d)}$ for some positive integer $d$;

(c) every number field contained in $K$ can be generated by elements of uniformly bounded degree

are equivalent. However, in general, we have that (드) implies (b), which implies

(国) and none of the inverse implications holds.

The work is structured in the following way.

Section 1 focuses on the study of the structure of the field $\mathbb{Q}^{(d)}$ and of Galois extensions with uniformly bounded local degrees. We prove that these are exactly those whose Galois group has finite exponent. Moreover, we prove that the existence of a uniform bound for the local degrees at almost every prime implies the uniform boundedness of the local degrees at every prime. The first part of the proof was already done in Checcoli and Zannier's paper [2, Remark 2, and it consists of an application of Chebotarev's density theorem; the second part is based on a theorem of Shafarevich on the number of generators of the Galois group of a $p$-extension of $p$-adic fields and on Zelmanov's result on the Restricted Burnside Problem. As already done in 2, Proposition 2.1, the case of abelian extensions is also discussed, proving the first part of Theorem 1. Explicit bounds for the local degrees are provided in some special cases.

In Section 2 we recall the main properties of extraspecial groups. These groups shall enable us to construct examples of fields entailing the validity of the statements of Theorem 1. We also describe some irreducible modules of extraspecial groups over finite fields which shall be used in our constructions. 
In Section 3 we prove the main theorem of this work on the nonequivalence of properties (a), (b) and (ㄷ), which is a generalization of Theorem 1.1 in [2]. The proof is based on some group-theoretical constructions due to A. Lucchini with extraspecial groups and their modules, followed by an application of Shafarevich's Theorem about the realization of solvable groups of odd order as Galois groups.

The use of Shafarevich's result can actually be avoided by realizing explicitly groups constructed in Section 3 and this will be done in Section 4 These constructions are based on some elementary methods in inverse Galois theory and on a result by Serre.

\section{Extensions With UNIFORMLY BOUNDED LOCAL DEGREES}

In this section we study some properties of the compositum of fields with bounded degrees, in order to give some motivations to our questions, and we provide a characterization of Galois extensions with uniformly bounded local degrees depending only on the exponent of their Galois group. Finally, we show the equivalence of conditions (国), (b) and (ㄷ) of Theorem 1 in the case of abelian extensions.

1.1. Galois extensions with finite exponent. We fix some notation. Let $d$ be a positive integer and let $F$ be a number field. We denote by $F^{(d)}$ the field obtained by taking the compositum of all algebraic extensions of $F$ of degree at most $d$ (over $F)$.

If $K$ is an infinite algebraic extension of $\mathbb{Q}$ and $p$ is a prime number, we define a valuation $v_{p}$ of $K$ which extends the $p$-adic valuation over $\mathbb{Q}$ in the following way. We denote by $\mathbb{Q}_{p}^{\text {alg }}$ an algebraic closure of $\mathbb{Q}_{p}$, by $\mathbb{C}_{p}$ its $p$-adic completion and by $w_{p}$ the unique valuation of $\mathbb{C}_{p}$ extending the $p$-adic valuation of $\mathbb{Q}$. Then we fix an embedding $\sigma: K \hookrightarrow \mathbb{C}_{p}$ and we define the valuation $v_{p}$ of $K$ as $v_{p}(x):=w_{p}(\sigma(x))$ for every $x \in K$.

The completion of $K$ with respect to $v_{p}$ is denoted by $K_{v_{p}}$ and it is an extension of $\mathbb{Q}_{p}$. We are interested in the case when its degree over $\mathbb{Q}_{p}$ is bounded, for every prime $p$ and every valuation $v_{p}$ above $p$, by a constant independent from $p$. We have the following definition.

Definition 1.1. An algebraic extension $K$ of $\mathbb{Q}$ has uniformly bounded local degrees if there exists a positive integer $B$ such that $\left[K_{v_{p}}: \mathbb{Q}_{p}\right]<B$ for every prime number $p$ and every valuation $v_{p}$ of $K$ extending the $p$-adic valuation of $\mathbb{Q}$.

One of the questions posed in this work is whether every algebraic extension of $\mathbb{Q}$ with uniformly bounded local degrees is contained in $\mathbb{Q}^{(d)}$ for some positive integer $d$. In order to give a motivation to this we recall that the field $\mathbb{Q}^{(d)}$ has uniformly bounded local degrees. We have the following proposition.

Proposition 1.1. Let $F$ be a number field of degree $n$ over $\mathbb{Q}$ and let $v$ be any valuation of $F$. Let $w$ be an extension of $v$ to $F^{(d)}$ and denote by $F_{w}^{(d)}$ and $F_{v}$ the completions of $F^{(d)}$ and $F$ with respect to $w$ and $v$ respectively. Then the local degree $\left[F_{w}^{(d)}: F_{v}\right]$ is bounded solely in terms of $n$ and $d$.

Proof. See for instance Proposition 1 of [1].

The next theorem is a generalization of Remark 2 of [2] and it provides a characterization of Galois extensions with uniformly bounded local degrees only in terms of the exponent of their Galois group. Moreover, it asserts that the existence of 
a uniform bound for the local degrees at almost every prime implies the uniform boundedness of the local degrees at every prime.

Theorem 1.2. Let $L$ be a number field and $K / L$ an infinite algebraic Galois extension. Then the following conditions are equivalent:

(1) $K$ has uniformly bounded local degrees at every prime of $L$;

(2) $K$ has uniformly bounded local degrees at almost every prime of $L$;

(3) $\operatorname{Gal}(K / L)$ has finite exponent.

Proof. Obviously (11) implies (2). Now we suppose that (2) holds; then $K$ has local degrees uniformly bounded by a constant $B$ at every prime except some primes belonging to a finite set $S$.

We fix a finite Galois extension $E$ of $L$ contained in $K$ and we take $\sigma \in \operatorname{Gal}(E / L)$. By Chebotarev's Density Theorem (see [7], Ch.7, §3, Theorem 7.30) there exists a prime $\wp$ of $L$ with $\wp \notin S$, a prime $\beta$ of $E$ unramified above $\wp$ and a conjugate $\tau$ of $\sigma$ that generates the decomposition group $D(\beta \mid \wp)$ which is cyclic and isomorphic to $\operatorname{Gal}\left(E_{\beta} / L_{\wp}\right)$, where $E_{\beta}$ and $L_{\wp}$ denote the completions of $E$ and $L$ with respect to $\beta$ and $\wp$ respectively. By assumption, $\left|\operatorname{Gal}\left(E_{\beta} / L_{\wp}\right)\right| \leq B$; thus $\sigma^{B !}=\tau^{B !}=$ $i d$ and $\exp (\operatorname{Gal}(E / L)) \leq B$ !. Since $\operatorname{Gal}(K / L)$ is the inverse limit of the family $\{\operatorname{Gal}(E / L)\}_{E}$, where $E$ varies among the finite Galois extension of $L$ contained in $K$, we have $\exp (G) \leq B$ ! and (3) holds.

Now we suppose that (3) holds for $K$ and we set $\exp (\operatorname{Gal}(K / L))=b$. We want to prove that the local degrees of $K$ are uniformly bounded at every prime.

We can write $K$ as the compositum of a family of number fields $\left\{K_{m}\right\}_{m}$, where $K_{m} / L$ is any finite Galois extension of $L$ contained in $K$ and we set $G_{m}=$ $\operatorname{Gal}\left(K_{m} / L\right)$.

We let $n=[L: \mathbb{Q}]$ and we fix a prime number $p$. For every $m$ we denote by $L_{v}$ and $K_{m, v}$ the completions of $L$ and $K_{m}$, respectively, with respect to any valuation $v$ extending the $p$-adic one. We recall that $\operatorname{Gal}\left(K_{m, v} / L_{v}\right)$, being isomorphic to a subgroup of $G_{m}$, has exponent at most $b$. We have to discuss three cases for the extension $K_{m, v} / L_{v}$.

If it is unramified, then it is cyclic of order bounded by $b$; if it is tamely ramified, then it is a metacyclic extension. Thus its order is bounded by $b^{2}$.

If it is wildly ramified, then the tamely ramified part always has degree at most $b^{2}$. The first ramification group of $K_{m, v} / L_{v}$ is a $p$-group of exponent at most $b$. Moreover, by a theorem of Shafarevich (see 9], Theorem 1) on the number of generators of the Galois group of a $p$-extension of $p$-adic fields, this group has at most $n b^{2}+2$ generators. So the problem reduces to the following: is it true that, for all positive integers $b$ and $m$, if a finite group $H$ has $m$ generators and exponent $b$, then the order of $H$ is bounded by a constant which depends only on $m$ and $b$ ?

This question is known as the Restricted Burnside Problem and was positively answered in 1989 by Efim Zelmanov; for a detailed description of Zelmanov's proof the interested reader should refer to [11]. In view of Zelmanov's result, the wildly ramified part of the extension has a degree which is bounded by a constant depending only on $n$ and $b$.

We can now conclude our proof. Summing up all the previous results, we get that for every $m$, the local degree $\left[K_{m, v}: \mathbb{Q}_{p}\right]$ is bounded by a constant depending only on $b$ and $n=[L: \mathbb{Q}]$, which are fixed. Thus the number of all possible completions of the $K_{m}$ at primes above $p$ is finite and independent from $p$. The compositum of this finite number of fields has degree over $\mathbb{Q}_{p}$ which is bounded by a constant 
independent of $p$ and it contains the completion of $K$ with respect to any valuation extending the $p$-adic one.

The proof of the previous result does not provide an explicit bound for the local degrees of an extension $K / \mathbb{Q}$ of finite exponent. In order to effectively compute such a bound, we need additional information on the order of the wild part of the completion of every finite Galois subextension of $K$. We have the following result.

Theorem 1.3. Let $K / \mathbb{Q}$ be an infinite Galois extension of exponent $b$ and let $\left\{K_{m}\right\}_{m}$ be the family of all finite Galois subextensions of $K$. Suppose that for every $m$, every prime number $p$ dividing $b$ and every valuation $v$ of $K_{m}$ above $p$ we have $v_{p}\left(\left|\operatorname{Gal}\left(K_{m, v} / \mathbb{Q}_{p}\right)\right|\right) \leq n$, where $v_{p}$ denotes the usual $p$-adic valuation of $\mathbb{Q}$.

Then $K$ has local degrees bounded by

$$
\prod_{i=1}^{n} A(i),
$$

where $A(1)=b^{3}$ and

$$
A(i+1)=b^{\left(\prod_{j=1}^{i} A(j)\right)+2} .
$$

Proof. We fix a prime number $p$ and we consider a valuation $w$ of $K$ extending the $p$-adic valuation of $\mathbb{Q}$. The field $K$ equals the compositum of all its finite Galois subextensions $\left\{K_{m}\right\}_{m}$ and, for every $m, v=\left.w\right|_{K_{m}}$ is a valuation of $K_{m}$ above $p$. We denote by $K_{w}$ and by $K_{m, v}$ the completions of $K$ and $K_{m}$ with respect to $w$ and $v$, respectively.

We fix $m$ and we set $G=\operatorname{Gal}\left(K_{m, v} / \mathbb{Q}_{p}\right)$. We consider the filtration of $G$ via its ramification subgroups

$$
G \triangleright G_{0} \triangleright G_{1} \triangleright G_{2} \triangleright \ldots \triangleright G_{r}=1,
$$

where:

- $G / G_{0}$ is cyclic being the Galois group of the unramified part of the extension $K_{m, v} / \mathbb{Q}_{p}$

- $G_{0} / G_{1}$ is the Galois group of the tame and totally ramified part of the extension and it is also cyclic;

- $G_{i} / G_{i+1}$ is $p$-elementary abelian for every $i \geq 1$.

If $p$ does not divide the exponent $b$, then the local extension can only be tamely ramified. The maximal unramified subextension of $K_{m, v}$ is the subfield of $K_{m, v}$ fixed by $G_{0}$ and we denote it by $K_{m, v}^{G_{0}}$. Then $K_{m, v}^{G_{0}} / \mathbb{Q}_{p}$ is contained in the compositum $L^{\mathrm{ur}, b}$ of all unramified extensions of $\mathbb{Q}_{p}$ of degree dividing $b$, which has degree $\left[L^{\mathrm{ur}, b}: \mathbb{Q}_{p}\right]=b$, since we have only one unramified extension of $\mathbb{Q}_{p}$ of every fixed degree. Therefore the maximal unramified subextension $K_{w}^{\mathrm{ur}}$ of $K_{w}$ is also contained in $L^{\mathrm{ur}, b}$.

We recall that from local class field theory (see 4, Theorem in Ch. IV, §3.3) there is a one-to-one correspondence between finite abelian extensions of a $p$-adic field $K$ and open subgroups of finite index of $K^{*}$. In particular this correspondence is such that if $L / K$ is the maximal abelian extension of $K$ of exponent $b$, then $\operatorname{Gal}(L / K) \simeq K^{*} /\left(K^{*}\right)^{b}$.

This applies to our situation, since the tame and totally ramified part of the extension $K_{m, v}^{G_{1}} / K_{m, v}^{G_{0}}$ is contained in the maximal abelian extension $L^{\text {tame,b}}$ of 
$L^{\mathrm{ur}, b}$ of exponent dividing $b$ and prime to $p$. Then we have

$$
\operatorname{Gal}\left(L^{\mathrm{tame}, b} / L^{\mathrm{ur}, b}\right) \simeq L^{\mathrm{ur}, b^{*}} /\left(L^{\mathrm{ur}, b^{*}}\right)^{a}
$$

for some integer $a$ prime to $p$ and smaller than $b$. Therefore

$$
\left|\operatorname{Gal}\left(L^{\mathrm{tame}, b} / L^{\mathrm{ur}, b}\right)\right| \leq|(\mathbb{Z} / a \mathbb{Z})|^{2} \leq b^{2} .
$$

Since this holds for every $m$, the maximal tame subextension $K_{w}^{\text {tame }}$ of $K_{w}$ is a subfield of $L^{\text {tame, } b}$.

If $p$ divides the exponent $b$, then one can have wild ramification. However, using the previous arguments, the degree over $\mathbb{Q}_{p}$ of the maximal tame subextension $K_{w}^{\text {tame }}$ of $K_{w}$ is bounded by $b^{3}$.

As for the wildly ramified part of the extension, since by hypothesis $v_{p}(|G|) \leq n$, the group $G_{1}$ has order at most $p^{n}$ and the filtration

$$
G_{1} \triangleright G_{2} \triangleright \ldots \triangleright G_{r}=1
$$

has at most $n$ jumps; this means that $G_{i} \neq G_{i+1}$ for at most $n$ indices $i$.

Therefore we have the following tower of fields:

$$
\mathbb{Q}_{p}=K_{m, v}^{G} \subseteq K_{m, v}^{G_{0}} \subseteq K_{m, v}^{G_{1}} \subseteq \ldots \subseteq K_{m, v}^{G_{r}}=K_{m, v}
$$

in which the extension $K_{m, v}^{G_{i+1}} / K_{m, v}^{G_{i}}$ is elementary $p$-abelian for at most $n$ indexes $i \geq 1$ and trivial otherwise.

We set $L_{1}=L^{\text {tame,b}}$ and we denote by $L_{i+1}$ the compositum of all $p$-elementary abelian extensions of $L_{i}$. We notice that $K_{m, v}^{G_{j}}$ is contained in some $L_{n}$ for every index $j$. Again from local class field theory, we have that

$$
\operatorname{Gal}\left(L_{i+1} / L_{i}\right)=\left(L_{i}^{*}\right) /\left(L_{i}^{*}\right)^{p} \simeq(\mathbb{Z} / p \mathbb{Z})^{\left[L_{i}: \mathbb{Q}_{p}\right]+\epsilon\left(L_{i}\right)},
$$

where $\epsilon\left(L_{i}\right)=1$ if $L_{i}$ does not contain $p$-th roots of unity and $\epsilon\left(L_{i}\right)=2$ otherwise. Since $p<b$ and $\left[L_{1}: \mathbb{Q}_{p}\right] \leq b^{3}=A(1)$, we have $\left[L_{2}: L_{1}\right] \leq b^{b^{3}+2}=A(2)$ and it is easy to see that $\left[L_{i}: L_{i-1}\right] \leq A(i)$. Since $K_{w}$ is contained in $L_{n}$, the result follows.

1.2. Abelian extensions. We consider infinite abelian extensions of $\mathbb{Q}$, namely infinite algebraic Galois extensions of $\mathbb{Q}$ with abelian (profinite) Galois group. The following theorem, which was already in 2], Proposition 2.1, gives an answer to our initial question for these extensions.

Theorem 1.4. For an infinite abelian extension $K$ of $\mathbb{Q}$, the following conditions are equivalent:

(a) K has uniformly bounded local degrees;

(b) there exists a positive integer d such that $K$ is contained in $\mathbb{Q}^{(d)}$;

(c) every finite abelian subextension of $K$ can be generated by elements of uniformly bounded degree.

Proof. It is trivial to prove that (ㄷ) implies (b). The fact that (b) implies (a) follows from Proposition 1.1 
We now assume that $K$ satisfies condition (国). We set $G=\operatorname{Gal}(K / \mathbb{Q})$; then, by Theorem $1.2 \exp (G) \leq b$ for some positive integer $b$ and

$$
G={\underset{m}{m}}_{\lim } G_{m}
$$

where $G_{m}=\operatorname{Gal}\left(K_{m} / \mathbb{Q}\right)$ and $K_{m}$ is any finite abelian extension of $\mathbb{Q}$ contained in $K$.

For every $m, G_{m}$ is a finite abelian group, and we can write it as a product of finite cyclic groups

$$
G_{m}=\prod_{i=1}^{n} U_{i}
$$

We let $H_{i}$ be the subgroup of $G_{m}$ defined as

$$
H_{i}:=\prod_{j \neq i} U_{j}
$$

We have $\left[G_{m}: H_{i}\right]=\left|U_{i}\right|=\exp \left(U_{i}\right) \leq b$ for all $i$ 's and $\bigcap_{i=1}^{n} H_{i}=1$.

Therefore $K_{m}$ is the compositum of the fields $\left\{K_{m}^{H_{i}}\right\}_{i}$ which satisfy the inequality $\left[K_{m}^{H_{i}}: \mathbb{Q}\right]=\left[G_{m}: H_{i}\right] \leq b$. Thus $K_{m}$ is generated by elements of uniformly bounded degree and $K$ satisfies condition (IC), which completes the proof.

Remark 1.5. We notice that an abelian extension of exponent $b$ has local degrees bounded by $b^{3}$. In fact if $K / \mathbb{Q}$ is an abelian extension of exponent $b$, the completion of $K$ with respect to a prime $p$ is contained in the maximal abelian extension $M$ of $\mathbb{Q}_{p}$ of exponent $b$; from local class field theory we have that

$$
\left|\operatorname{Gal}\left(M / \mathbb{Q}_{p}\right)\right|=\left|\mathbb{Q}_{p}{ }^{*} /\left(\mathbb{Q}_{p}{ }^{*}\right)^{b}\right| \leq b^{3} .
$$

\section{EXtraspecial GROUPS}

In this section we recall definitions and properties of extraspecial groups and their irreducible modules which shall be used later in our constructions. A detailed discussion on extraspecial groups and related topics can be found in 3 .

\subsection{Definition and properties.}

Definition 2.1. Let $p$ be a prime. A $p$-group $G$ is said to be extraspecial if the center $Z(G)$ and the commutator subgroup $G^{\prime}$ are equal and have order $p$.

We recall the main properties of extraspecial groups which shall be used in our constructions.

Proposition 2.1. For every odd prime $p$ and every positive integer $m$ there exists an extraspecial group $G_{m}$ with the following properties:

(1) $G_{m}$ has order $p^{2 m+1}$ and exponent $p$;

(2) the quotient $G_{m} / Z\left(G_{m}\right)$ is elementary abelian of order $p^{2 m}$;

(3) $G_{m}$ is isomorphic to $\left(E_{1} \times \ldots \times E_{m}\right) / N_{m}$, where $E_{1}, \ldots, E_{m}$ are extraspecial groups of order $p^{3}$ and exponent $p$, the center of $E_{i}$ is generated by an element $z_{i}$ and

$$
N_{m}=\left\{\left(z_{1}^{a_{1}}, \ldots, z_{n}^{a_{n}}\right) \mid \sum_{i=1}^{n} a_{i} \equiv 0 \quad \bmod p\right\} ;
$$

(4) every abelian subgroup of $G_{m}$ has order smaller than $p^{m+1}$. 
Moreover, every extraspecial group of order $p^{2 m+1}$ and exponent $p$ satisfies all the above properties and it is unique up to isomorphisms.

Proof. See [3], Chap. A, $\S 19$ and $\S 20$.

Remark 2.2. It is easy to prove that every nonabelian group of order $p^{3}$ is extraspecial and that, if $p$ is an odd prime, there exists a unique extraspecial group of order $p^{3}$ and exponent $p$, up to isomorphisms, namely the Heisenberg group with the presentation

$$
H=\left\langle x, y \mid x^{p}=y^{p}=1,[x, y] \in Z(H)\right\rangle .
$$

2.2. Some irreducible modules of an extraspecial group. We now want to give a description of certain irreducible modules of an extraspecial $p$-group over a finite field which shall be used to construct examples in the next section.

We fix two odd primes $p$ and $q$, with $p$ dividing $q-1$, such that the finite field $\mathbb{F}_{q}$ contains a primitive $p$-th root of unity $\zeta$. We consider the extraspecial group $H$ of order $p^{3}$, exponent $p$ and generators $x$ and $y$ and we set $W=\left(\mathbb{F}_{q}\right)^{p}$. Then $W$ has the structure of an $H$-module via the following action: if $\left\{e_{1}, \ldots, e_{p}\right\}$ is a basis for $W$, then $x$ permutes these elements as a cycle of length $p$ and $y \cdot e_{i}=\zeta^{i} e_{i}$.

Now for every positive integer $m$ we denote by $G_{m}$ the extraspecial group of order $p^{2 m+1}$ and exponent $p$ satisfying Proposition 2.1 and by $W_{m}$ the tensor product of $m$ copies of $W$. Then $G_{m}$ is isomorphic to a quotient of the direct product of $m$ copies of $H$; thus it acts on $W_{m}$, which can be regarded as a $G_{m}$-module of dimension $p^{m}$ over $\mathbb{F}_{q}$.

We recall the following definition:

Definition 2.3. If $G$ is a group and $M$ is a $G$-module over a field $k$, then $M$ is said to be irreducible (or simple) if it does not have proper nonzero $G$ submodules.

A field $k$ is said to be a splitting field for a group $G$ if every irreducible $G$-module remains irreducible over every extension of $k$.

We have the following proposition.

Proposition 2.2. $W_{m}$ is a faithful and irreducible $G_{m}$-module.

Proof. We first prove that the module $W_{m}$ is faithful for $G_{m}$. In fact suppose that there exists $g \in G_{m}, g \neq 1$, such that $g \cdot w=0$ for every $w \in W_{m}$. We have that, by construction,

$$
G_{m} \simeq(H \times \ldots \times H) / N,
$$

where $N$ is the normal subgroup of $H \times \ldots \times H$ described in Proposition 2.1(3); then $g=\left[\left(g_{1}, \ldots, g_{m}\right)\right]_{N}$ with $g_{i} \in H$ is a certain class of a quotient of the direct product of $m$ copies of $H$. We consider a basis $\left\{e_{1}, \ldots, e_{p}\right\}$ for $W$ and we choose $w=e_{1} \otimes 1 \otimes \ldots \otimes 1$. Then $g \cdot w=\zeta e_{i} \otimes 1 \otimes \ldots \otimes 1$ where $\zeta$ is some $p$-th root of unity. Then $g \cdot w \neq 0$, which is a contradiction.

Now we want to prove that $W_{m}$ is an irreducible $G_{m}$-module. To do this, we first show that $W$ is an irreducible $H$-module. Again we fix a basis $\mathcal{B}=\left\{e_{1}, \ldots, e_{p}\right\}$ for $W$ and we suppose that $M$ is a nonzero $H$-submodule of $W$. We may assume that $M$ is cyclic; this means that there exists an element $w=a_{1} e_{1}+\ldots+a_{p} e_{p} \in W$ generating $M$. We define the elements $w_{1}, \ldots, w_{p-1}$ in the following way: $w_{1}:=w$ and $w_{i}:=x \cdot\left(w_{i-1}-y \cdot w_{i-1}\right)$. We notice that $w_{i} \in M$ for every $i$ and that the 
first $i$ coordinates of $w_{i}$ in the base $\mathcal{B}$ are zero. It follows that $w_{p-1}$ is a multiple of $e_{p}$; therefore $M$ contains $e_{p}$ and, since $x^{i} \cdot e_{p}=e_{i}$ belongs to $M$ for every $i$, then $M=W$.

Now, from Corollary 5.21 of $\left[3\right.$, we have that $\mathbb{F}_{q}$ is a splitting field for $H$ (since $H$ has exponent $p$ and $\mathbb{F}_{q}$ contains a $p$-th root of unity); in this case, using Theorem 5.22 of [3], we conclude that the tensor product of $m$ copies of $W$ is an irreducible representation of $H^{m}=H \times \ldots \times H$ with kernel the subgroup $N$ introduced above. Therefore it is also an irreducible $G_{m}$-module.

We end this section with some properties of an extraspecial group. These results seem to be ad hoc as they will be used only at the end of the next section, when their importance shall become evident.

The first result is about the cardinality of subgroups of extraspecial groups with a bounded number of generators. This will be used to provide an explicit bound for the local degrees of a certain infinite algebraic extension of the rationals, which we shall construct in the next section.

Proposition 2.3. Let $G$ be an extraspecial group of order $p^{2 m+1}$ and let $H$ be a subgroup of $G$ with at most $n$ generators. Then $|H| \leq p^{n+1}$.

Proof. We suppose first that $H$ contains the center $Z(G)$. Then we have

$$
H / Z(G) \subseteq G / Z(G) \simeq(\mathbb{Z} / p \mathbb{Z})^{2 m}
$$

and $H / Z(G)$ has at most $n$ generators as a subspace of $G / Z(G)$. Hence $|H / Z(G)| \leq$ $p^{n}$, since it is elementary abelian as a group, and $|H| \leq p^{n+1}$.

Now we suppose that $Z(G) \nsubseteq H$ and we consider $K=H Z(G)$, which is a subgroup of $G$ (since $Z(G)$ is normal in $G$ ) that contains the center and has at most $n+1$ generators ( $n$ generators from $H$ and one generator from the center which is cyclic by hypothesis). With the previous argument we obtain that $|K / Z(G)| \leq p^{n+1}$ and $|K| \leq p^{n+2}$. Finally, we get $|H| \leq p^{n+1}$.

The second property is about the intersection of all subgroups of an extraspecial group of bounded index. It will be used to provide subfields of $\mathbb{Q}^{\left(p^{3}\right)}$ which cannot be generated by elements of uniformly bounded degree.

Proposition 2.4. Let $p$ be an odd prime and $G$ be an extraspecial p-group of order $p^{2 m+1}$. Then the intersection of all subgroups of $G$ of index at most $p^{m}$ contains $Z(G)$.

Proof. Let $H$ be a subgroup of $G$ such that $[G: H] \leq p^{m}$; we have that $|H| \geq p^{m+1}$. Either $H$ contains the center $Z(G)$ or $|H Z(G)|>p^{m+1}$ and from Proposition 2.1 . $H Z(G)$ is not abelian; therefore $H$ is also not abelian and it contains two elements which do not commute. This implies that $G^{\prime}=Z(G) \subseteq H$, since $G^{\prime}$ is cyclic of order $p$.

\section{THE MAIN THEOREM}

In Section 1 we proved that for an infinite abelian extension of $\mathbb{Q}$ there is a list of properties which are equivalent to the property of having uniformly bounded local degrees. We now want to prove that this is not true in general. The nonequivalence is entailed by the existence of fields constructed using extraspecial groups and their modules. We now state the main result, which will be proved throughout this section and which provides a significant generalization of Theorem 1.1 of [2]. 
Theorem 2. Let $K$ be an infinite algebraic Galois extension of $\mathbb{Q}$. Consider the following properties for $K$ :

(a) K has uniformly bounded local degrees at almost every prime;

(b) $K$ is contained in $\mathbb{Q}^{(d)}$ for some positive integer $d$;

(c) every number field contained in $K$ can be generated by elements of uniformly bounded degree over $\mathbb{Q}$;

(d) there exists a positive integer $b$ and a family $\left\{K_{m}\right\}_{m}$ of finite Galois extensions of $\mathbb{Q}$ such that $K$ is the compositum of the family and for every $m$ the Galois group of $K_{m}$ has only minimal normal subgroups of order at most $b$.

Then:

(1) $($ (c) $) \Rightarrow($ b $) \Rightarrow($ (a) $)$;

(2) $(\mathrm{b}) \Rightarrow(\mathrm{d})$.

However no other implication holds, that is:

(i) (d) $\nRightarrow($ (b);

(ii) $(\mathrm{b}) \neq($ (c) ;

(iii) (a) $\nRightarrow($ (b) .

Remark 3.1. It is immediate to see that if $K$ satisfies (IC), then it also satisfies (b) and, from Theorem 1.1, it follows easily that ( $(\mathbf{c}) \Rightarrow(\underline{b}) \Rightarrow($ a $)$.

As the proof of the result is quite long, we divide it into four different parts.

3.1. Proof of (2). We want to prove that (b) implies (d). In order to do this we need the following result, which is Proposition 3.2 of [2]. We restate and reprove it here for clarity.

Lemma 3.2. Let $G$ be a finite group with a minimal normal subgroup $W$ such that $|W|=m$. Suppose that $G$ is a quotient of a group $H$, where $H$ is a subgroup of a direct product $H_{1} \times \ldots \times H_{s}$. Then $\left|H_{i}\right| \geq m$ for some index $i \in\{1, \ldots, s\}$.

Proof. We suppose that $G=H / N$ with $H \leq H_{1} \times \ldots \times H_{s}$ and that $\left|H_{i}\right|<m$ for every $i$. Let us denote by $\pi: H \rightarrow H / N$ the projection map. We set $\overline{H_{i}}:=$ $H \cap 1 \times \ldots \times 1 \times H_{i} \times \ldots \times H_{s}$ and we denote by $G_{i}=\pi\left(\overline{H_{i}}\right)$ the image of $\overline{H_{i}}$ in $G$. We notice that the $G_{i}$ 's are all normal subgroups of $G$.

We want to show by induction that $W \subseteq G_{i}$ for every $i$.

For $i=1$ this holds by assumption, since $G_{1}=G$.

Suppose it is true for $G_{i-1}$. Now $W \subseteq G_{i-1}$ and $\left[G_{i-1}: G_{i}\right]<m$; thus $U:=$ $G_{i} \cap W$ is normal and nontrivial and so it contains $W$. Therefore $W \subseteq G_{i}$. In particular $W \subseteq G_{s}$, which is a contradiction, since $\left|G_{s}\right| \leq\left|H_{s}\right|<m$.

Now we are able to prove our result. We suppose that $K \subseteq \mathbb{Q}^{(d)}$ for some integer $d$. We write $K$ as the compositum of all its finite Galois subextensions $\left\{K_{m}\right\}_{m}$ and we set $G_{m}=\operatorname{Gal}\left(K_{m} / \mathbb{Q}\right)$. We suppose, moreover, that $K$ does not satisfy property (d). This means that there exists an infinite subsequence $N$ of the positive integers and a strictly increasing sequence of positive integers $\left\{c_{m}\right\}_{m \in N}$ such that $G_{m}$ has a minimal normal subgroup of order $c_{m}$ for every $m \in N$.

Thus, in view of Lemma 3.2, whenever $G_{m}$ is isomorphic to a quotient $H / N$ and $H$ is a subgroup of a direct product $H_{1} \times \ldots \times H_{s}$ and $m \in N$, then $\left|H_{i}\right| \geq c_{m}$ for at least one index $i$. 
We fix $m \in N$ such that $d !<c_{m}$. We notice that, since $K$ is contained in $\mathbb{Q}^{(d)}$, so is $K_{m}$; then there exist number fields $L_{1}, \ldots, L_{s}$ such that $K_{m} \subseteq L_{1} \ldots L_{s}$ and $\left[L_{i}: \mathbb{Q}\right] \leq d$.

We denote by $L_{i}^{\text {Gal }}$ the Galois closure of $L_{i}$ in $\mathbb{Q}^{\text {alg }}$ and by $H_{i}=\operatorname{Gal}\left(L_{i}^{\text {Gal }} / \mathbb{Q}\right)$ its Galois group. We notice that $\left|H_{i}\right| \leq d$ ! $<c_{m}$ by hypothesis.

We have $K_{m} \subseteq L_{1}^{\mathrm{Gal}} \ldots L_{s}^{\mathrm{Gal}}$ and $\operatorname{Gal}\left(L_{1}^{\mathrm{Gal}} \ldots L_{s}^{\mathrm{Gal}}\right)$ is isomorphic to a subgroup of $H_{1} \times \ldots \times H_{s}$ via the restriction map.

Since $K_{m}$ is a Galois extension,

$$
G_{m}=\operatorname{Gal}\left(K_{m} / \mathbb{Q}\right) \simeq \operatorname{Gal}\left(L_{1}^{\mathrm{Gal}} \ldots L_{s}^{\mathrm{Gal}}\right) / N
$$

for some normal subgroup $N$ of $\operatorname{Gal}\left(L_{1}^{\mathrm{Gal}} \ldots L_{s}^{\mathrm{Gal}}\right)$.

Now $\operatorname{Gal}\left(L_{1}^{\mathrm{Gal}} \ldots L_{s}^{\mathrm{Gal}}\right)$ is a subgroup of $\left(H_{1} \times \ldots \times H_{s}\right)$ with $\left|H_{i}\right| \leq c_{m}$ for every $i$, which is a contradiction. Thus $K$ satisfies (d).

3.2. Proof of (i). We have to prove that condition (d) does not imply condition (b). In order to do this we fix a prime $p$ and we consider a family $\left\{K_{m}\right\}_{m}$ of finite Galois extensions of $\mathbb{Q}$ such that $G_{m}=\operatorname{Gal}\left(K_{m} / \mathbb{Q}\right)=\mathbb{Z} / p^{m} \mathbb{Z}$ is the cyclic group of order $p^{m}$. We denote by $K$ the compositum of all the $K_{m}$.

We notice that $\exp (\operatorname{Gal}(K / \mathbb{Q}))$ is not finite, since the group $\operatorname{Gal}(K / \mathbb{Q})$ contains elements of order $p^{m}$ for every $m$. In view of Theorem 1.2, $K$ cannot have uniformly bounded local degrees and therefore it is not contained in $\mathbb{Q}^{(d)}$.

However, for every $m$, every minimal normal subgroup of $G_{m}$ has order $p$ and property (d) holds.

3.3. Proof of (ii). We want to show that condition (b) does not imply condition (IC). We fix an odd prime number $p$ and we now consider an infinite family $\mathcal{F}=\left\{F_{i}\right\}_{i}$ of linearly disjoint Galois extensions of $\mathbb{Q}$ (for the definition of a linearly disjoint family of extensions, see [5], Ch. 2, $§ 2.5)$, where, for every $i, \operatorname{Gal}\left(F_{i} / \mathbb{Q}\right)=H$ is the extraspecial group of order $p^{3}$ and exponent $p$.

We know that such a family exists: in fact a famous theorem of Shafarevich (see [10]) asserts that every solvable group of odd order can be realized over $\mathbb{Q}$. Now, for every integer $n$, the group $H^{n}=H \times \ldots \times H$ is solvable and hence realizable. We denote by $F$ its realization, that is, $F$ is a Galois extension of $\mathbb{Q}$ with $\operatorname{Gal}(F / \mathbb{Q})=H^{n}$. We notice that $H^{n}$ has $n$ normal subgroups $H_{1}, \ldots, H_{n}$ isomorphic to $H^{n-1}$ whose intersection is trivial and $H^{n} / H_{i} \simeq H$ for all $i$ 's. Thus $F^{H_{1}}, \ldots, F^{H_{n}}$ are $n$ linearly disjoint realizations of $H$ over $\mathbb{Q}$ and we can make this construction for every integer $n$.

Now we consider the family of finite Galois extensions $\left\{K_{m}\right\}_{m}$ constructed in the following way: for every $m$, denote by $L_{m}=F_{1} \cdot \ldots \cdot F_{m}$ the compositum of $m$ fields of the family $\mathcal{F}$. By construction

$$
\operatorname{Gal}\left(L_{m} / \mathbb{Q}\right)=H \times \ldots \times H=H^{m} .
$$

Next note that the subgroup $N_{m}$ of $H^{m}$ described in Proposition 2.1 is normal and that $H^{m} / N_{m}$ is extraspecial of order $p^{2 m+1}$. We denote by $K_{m}:=L_{m}^{N_{m}}$ the subfield of $L_{m}$ fixed by this subgroup. We have that $K_{m} / \mathbb{Q}$ is a finite Galois extension with Galois group an extraspecial group of order $p^{2 m+1}$ and exponent $p$.

We denote by $K$ the compositum of all the fields $\left\{K_{m}\right\}_{m}$. Since by construction every field $K_{m}$ is contained in $\mathbb{Q}^{\left(p^{3}\right)}$, therefore $K \subseteq \mathbb{Q}^{\left(p^{3}\right)}$ and (b) holds for $K$. 
However, $K$ does not satisfy (ㄷ). In fact suppose that every $K_{m}$ can be generated by elements of degree smaller than $d$, that is, $K_{m}$ equals the compositum of all its subextensions of degree at most $d$ over $\mathbb{Q}$.

We fix $m$ such that $d<p^{m}$ and we denote by $\left\{H_{m, i}\right\}_{i}$ the family of all subgroups of $G_{m}=\operatorname{Gal}\left(K_{m} / \mathbb{Q}\right)$ of index smaller than $c_{m}:=p^{m}$ in $G_{m}$. Then, by the Galois correspondence, $\left\{K_{m}^{H_{m, i}}\right\}_{i}$ is the family of all subextensions of $K_{m}$ of degree smaller than $c_{m}$ over $\mathbb{Q}$. We notice that the compositum $R_{m}:=\prod_{i} K_{m}^{H_{m, i}}$ is strictly contained in $K_{m}$ since, by Proposition 2.4, we have

$$
Z\left(G_{m}\right) \subseteq \bigcap_{i} H_{m, i}=\operatorname{Gal}\left(K_{m} / R_{m}\right) \neq 1
$$

This contradicts the hypothesis on $K_{m}$.

Remark 3.3. To avoid the use of Shafarevich's result, we give, in Section 4, an explicit realization of the family $\mathcal{F}$ and of the fields $K_{m}$.

3.4. Proof of (iii). We now want to prove that (a) does not imply (b); that is, we have to show the existence of an extension $K / \mathbb{Q}$ with uniformly bounded local degrees such that $K$ is not contained in $\mathbb{Q}^{(d)}$ for any integer $d$. This was already proved in 2, Theorem 1.1, and we recall here this proof.

In view of Theorem 1.2 and property (2), in order to construct such a field, it suffices to find a family of finite Galois extensions $\left\{K_{m}\right\}_{m}$ such that, setting $G_{m}:=\operatorname{Gal}\left(K_{m} / \mathbb{Q}\right)$, the family of groups $\left\{G_{m}\right\}_{m}$ satisfies the following conditions:

(1) $\exp \left(G_{m}\right)$ is bounded by a constant which does not depend on $m$;

(2) there exists a strictly increasing sequence of positive integers $\left\{c_{m}\right\}_{m}$ such that $G_{m}$ has a minimal normal subgroup of order $c_{m}$.

Then the compositum $K$ of the fields $K_{m}$ has the desired property.

In fact condition (11) implies that $\exp (\operatorname{Gal}(K / \mathbb{Q}))$ is finite, which is equivalent to the fact that $K$ has uniformly bounded local degrees.

As for condition (2), it implies that (b) does not hold for $K$. The proof of this fact is very similar to that of $(d \mathrm{~d}) \nRightarrow(\mathrm{b})$, even if condition (2) is not equivalent to condition (d). Suppose that (2) holds and that $K \subseteq \mathbb{Q}^{(d)}$ for some $d$. Then choose $m$ such that $d !<c_{m}$. Then $K_{m} \subseteq \mathbb{Q}^{(d)}$. From previous considerations this implies that $G_{m}$ is isomorphic to the quotient of a direct product of groups of order bounded by $d$ !. But since (2) holds we get a contradiction applying Lemma 3.2

The construction of $K$ goes as follows.

We take $p$ and $q$ to be two odd primes such that $p$ divides $q-1$. We denote by $E_{m}$ the extraspecial group of order $p^{2 m+1}$ and exponent $p$ satisfying Proposition 2.1 and by $\mathbb{F}_{q}\left[E_{m}\right]$ its regular representation, that is,

$$
\mathbb{F}_{q}\left[E_{m}\right]=\left\{\sum_{g \in E_{m}} a_{g} g \mid a_{g} \in \mathbb{F}_{q}\right\}
$$

Now we consider the family of finite groups $\left\{G_{m}\right\}_{m \geq 1}$, where $G_{m}=\mathbb{F}_{q}\left[E_{m}\right] \rtimes E_{m}$ and the semidirect product is taken via the natural action by translation of $E_{m}$ on $\mathbb{F}_{q}\left[E_{m}\right]$. We can now prove the following lemma.

Lemma 3.4. The family $\left\{G_{m}\right\}_{m \geq 1}$ satisfies condition (11) with bound $p q$ and condition (2) with $c_{m}=q^{p^{m}}$. 
Proof. Since $\exp \left(G_{m}\right)=p q$ for every $m$, condition (11) holds.

As for condition (2), we consider the irreducible $E_{m}$-module $W_{m}$ constructed in Section 2.2. Since $q$ and $p$ are different primes, by Maschke's theorem (see [3], Ch. A, $\S 11$, Thm. 11.5), the group algebra $\mathbb{F}_{q}\left[E_{m}\right]$ is a semisimple algebra. In particular the irreducible $E_{m}$-module $W_{m}$ appears as a direct summand of $\mathbb{F}_{q}\left[E_{m}\right]$. By abuse of notation, we denote by $W_{m}$ a subgroup of $\mathbb{F}_{q}\left[E_{m}\right]$ isomorphic to $W_{m}$ and by $H_{m}$ the subgroup of $G_{m}$ given by $H_{m}:=W_{m} \rtimes 1$.

Then the subgroup $H_{m}$ is a normal subgroup of $G_{m}$ and it is also minimal, being isomorphic to $W_{m}$, which is an irreducible module. Moreover, it has order $\left|H_{m}\right|=q^{p^{m}}$, and therefore condition (2) is true with $c_{m}=\left|H_{m}\right|=q^{p^{m}}$.

Now, having constructed a family of groups which satisfy the desired properties, we want to find a family of number fields realizing these groups.

It is easy to show that such a family exists: in fact, the groups $G_{m}$ are $p q$ groups and, by Burnside's Theorem (see [3], Ch. I, §2, p. 210), they are solvable. Therefore, in view of Shafarevich's Theorem (see [10]), they can be realized over $\mathbb{Q}$ (recall that $p$ and $q$ are odd primes); that is, there exists a family of number fields $\left\{K_{m}\right\}_{m \geq 1}$ such that, for every $m, \operatorname{Gal}\left(K_{m} / \mathbb{Q}\right) \simeq G_{m}$ and we denote by $K$ the compositum of this family. Then $K$ satisfies (国), but not (b) of Theorem 2 .

Remark 3.5. Shafarevich's result is not necessary to prove the existence of such a family, since this will be constructed explicitly in Section 4.2 .

Even if Theorem 1.2 is sufficient to prove that the constructed field $K$ has uniformly bounded local degrees, one may be interested in knowing an effective bound for the local degrees of $K$.

We fix a prime number $\ell$ and we consider the completion of every $K_{m}$ at a prime $v$ above $\ell$, say $K_{m, v}$. The Galois group of the tame part of the extension $K_{m, v} / \mathbb{Q}_{\ell}$ is metabelian of order at most $(p q)^{3}$. The first ramification group $G_{1}$ of the local extension has the following structure:

- for $\ell=q$ it is a subgroup of $\mathbb{F}_{q}\left[E_{m}\right]$ and therefore elementary abelian of order at most $q^{(p q)^{3}+2}$;

- for $\ell=p$ it is a subgroup of $E_{m}$ with at most $(p q)^{3}+2$ generators. In view of Proposition 2.3. $\left|G_{1}\right| \leq p^{(p q)^{3}+3}$.

- $G_{1}$ is trivial otherwise.

Now we can apply the formula given in Theorem 1.3 with $n=(p q)^{3}+3$ and we obtain an explicit bound for the local degrees of $K$.

However this bound can be significantly improved via the following considerations. We again fix a prime $\ell$ of $\mathbb{Q}$ and we denote by $v$ a valuation of $K_{m}$ above $\ell$. We have to discuss three cases.

(1) If $\ell \neq p, q$, then, for every $m$, the extension $K_{m, v} / \mathbb{Q}_{\ell}$ is tame and it is contained in the compositum of all metabelian extensions of $\mathbb{Q}_{\ell}$ of exponent dividing $p q$, which has degree bounded by $q^{6}$.

Then the local degrees of $K$ at prime $\ell \neq p, q$ are bounded by $q^{6}$.

(2) If $\ell=q$, the unramified part of the extension is contained in the compositum $L^{\text {ur }}$ of all unramified extensions of $\mathbb{Q}_{q}$ of degree dividing $p q$ and this extension has degree bounded by $p q$. 
The tame and totally ramified part of the extension is either trivial or cyclic of degree $p$. Therefore it is contained in the compositum $L^{\text {tame }}$ of all cyclic extensions of $L^{\mathrm{ur}}$ of degree $p$, which has degree bounded again by $p^{2}$.

The wild ramified part, having Galois group which is a subgroup of $\mathbb{F}_{q}\left[E_{m}\right]$ and therefore elementary abelian, is contained in the compositum of all $q$-elementary abelian extensions of $L^{\text {tame }}$, which has degree over $L^{\text {tame }}$ at most $q^{p^{3} q+2}$.

Therefore the local degree of $K$ at $q$ is at most $q^{p^{3} q+3} p^{3}<q^{q^{4}+6}$.

(3) If $\ell=p$ we denote by $H_{m}$ the Galois group of the local extension $K_{m, v} / \mathbb{Q}_{p}$. Since $\operatorname{Gal}\left(K_{m} / \mathbb{Q}\right)=\mathbb{F}_{q}\left[E_{m}\right] \rtimes E_{m}$ is a $p q$-group with a unique $q$-Sylow subgroup $\mathbb{F}_{q}\left[E_{m}\right]$, which is normal, then $H_{m}$ also possesses a unique $q$ Sylow subgroup $Q_{m}$.

By Schur-Zassenhaus' theorem (see [3], Ch. A, §11, Theorem 11.3), since $p$ and $q$ are different primes, $H_{m}=Q_{m} \rtimes P_{m}$, where $P_{m}$ is the $p$-Sylow subgroup, $Q_{m} \leq \mathbb{F}_{q}\left[E_{m}\right], P_{m} \leq E_{m}$ and the action of $P_{m}$ on $Q_{m}$ is induced by the action of $E_{m}$ on $\mathbb{F}_{q}\left[E_{m}\right]$ by restriction.

We denote by $K_{m, v}^{Q_{m}}$ the subfield of $K_{m, v}$ fixed by $Q_{m}$. Then the extension $K_{m, v}^{Q_{m}} / \mathbb{Q}_{p}$, having metabelian Galois group $P_{m}$, is contained in the compositum $L$ of all metabelian $p$-extensions of $\mathbb{Q}_{p}$, which has degree $\left[L: \mathbb{Q}_{p}\right] \leq p^{p^{2}+4} \leq q^{q^{2}+4}$.

The extension $K_{m, v} / K_{m, v}^{Q_{m}}$ is an abelian $q$-extension of a $p$-adic field with $(p, q)=1$; thus it is contained in the compositum $L^{\prime}$ of all tamely ramified extensions of $L$ of degree $q$ and we have $\left[L^{\prime}: L\right] \leq q^{2}$.

Then the local degree of $K$ at $p$ is bounded by $q^{q^{2}+6}$.

Summing up these results, $K$ has local degrees bounded by $q^{q^{4}+6}$.

\section{Explicit CONStructions}

In Section 3 we saw that the existence of certain Galois extensions with special properties was among the basic ingredients of the proof of our main theorem; this was done by using Shafarevich's Theorem on the realizability of solvable groups of odd order over $\mathbb{Q}$.

This section provides explicit realizations of those groups. These constructions are interesting for two reasons: first, they permit us to avoid the use of Shafarevich's result. Secondly, coming back to one of the original motivations of this work, it is proved in 12 that the Northcott property for an infinite algebraic extension $K$ of $\mathbb{Q}$ is strictly related to the behavior of the discriminants of certain finite subextensions of $K$. Therefore, knowing how to concretely construct fields described in Section 3 could be a step towards understanding whether these extensions might have the Northcott property or not.

Section 4.1 is devoted to describing a method, based on some results in [6, to realize extraspecial $p$-groups over a field of characteristic zero containing a primitive $p$-th root of unity. This entails the existence of the family of number fields used in the proof of (iii) of Theorem 2, as pointed out in Remark 3.3. In Section 4.2 we use a method described by Serre in [8] to solve embedding problems with abelian kernel. This result, together with the construction of Section 4.1, provides a realization of the family of Galois extensions needed to prove part (iii) of the main theorem, as announced in Remark 3.5 . 
4.1. Realizing extraspecial $p$-groups of exponent $p$. In this section we generalize a method described in [6] which can be used to realize extraspecial $p$-groups of exponent $p$ over fields of characteristic zero containing a primitive $p$-th root of unity. We have the following theorem.

Theorem 4.1. Let $k$ be a field of characteristic zero containing a primitive $p$-th root of unity $\zeta_{p}$. Let $a_{1}, b_{1}, \ldots, a_{m}, b_{m} \in k$ be elements chosen in the following way.

(i) For every $i$ the element $a_{i}$ is not a $p$-th power in $k$ and the ideal $\left(a_{i}\right)$ is prime to the ideals $\left(a_{j}\right)$ and $\left(b_{j}\right)$ for every $j<i$.

(ii) Denote by $\sigma_{i}$ the generator of $\operatorname{Gal}\left(k\left(\sqrt[p]{a_{i}}\right) / k\right)$ such that $\sigma_{i}\left(\sqrt[p]{a_{i}}\right)=\zeta_{p} \sqrt[p]{a_{i}}$. Let $\mathfrak{q}_{i}$ be a prime of $k$ which splits completely in the extension $k\left(\sqrt[p]{a_{i}}\right) / k$ and such that $\mathfrak{q}_{i} \neq \mathfrak{q}_{j}$ for every $j<i$.

Denote by $\beta_{1}^{i}, \ldots, \beta_{p}^{i}$ the primes of $k\left(\sqrt[p]{a_{i}}\right)$ above $\mathfrak{q}_{i}$ and by $S_{i}$ the set of primes of $k\left(\sqrt[p]{a_{i}}\right)$ lying above the primes of $k$ dividing $\left(a_{j}\right)$ or $\left(b_{j}\right)$ for $j<i$. Let $x_{i} \in k\left(\sqrt[p]{a_{i}}\right)$ be a solution of the system

$$
\begin{cases}v_{\beta_{1}^{i}}(X)=1 & \\ v_{\beta_{j}^{i}}(X)=0 & \forall j \neq 1 \\ v_{\beta}(X)=0 & \forall \beta \in S_{i}\end{cases}
$$

and set $b_{i}:=N_{\sigma_{i}}\left(x_{i}\right)$, where $N_{\sigma_{i}}$ is the norm function of the extension $\left.k\left(\sqrt[p]{(} a_{i}\right)\right) / k$.

Finally, consider the elements $\omega_{1}, \ldots, \omega_{m}$ defined as

$$
\omega_{i}:=\frac{b_{i}^{p-1}}{\prod_{j=i}^{p-1} \sigma_{i}^{j}\left(x_{i}^{j}\right)} .
$$

Then:

(a) $\left.\left\{k\left(\sqrt[p]{a_{i}}, \sqrt[p]{b_{i}}, \sqrt[p]{\omega_{i}}\right)\right)\right\}_{i}$ is a family of linearly disjoint Galois extensions of $k$ and, for every index $i$, the group $\left.H_{i}:=\operatorname{Gal}\left(k\left(\sqrt[p]{a_{i}}, \sqrt[p]{b_{i}}, \sqrt[p]{\omega_{i}}\right)\right) / k\right)$ is isomorphic to the extraspecial group of order $p^{3}$ and exponent $p$;

(b) the extension $k\left(\sqrt[p]{a_{1}}, \sqrt[p]{b_{1}}, \ldots, \sqrt[p]{a_{m}}, \sqrt[p]{b_{m}}, \sqrt[p]{\omega_{1} \ldots \omega_{m}}\right) / k$ is a Galois extension with Galois group the extraspecial group of order $p^{2 m+1}$ and exponent $p$.

Proof. The first part of property (国) follows from the results of [6]; in fact, setting

$$
M_{i}:=k\left(\sqrt[p]{a_{i}}, \sqrt[p]{b_{i}}, \sqrt[p]{\omega_{i}}\right),
$$

it is proved in [6] that the extensions $M_{i} / k$ are Galois extensions with Galois group $H_{i}$ which is an extraspecial group of order $p^{3}$ and exponent $p$.

We want to prove that the extensions $M_{i}$ are linearly disjoint and that property (b) holds. We prove the result by induction on $m$. The theorem is trivially true for $m=1$.

We suppose the theorem to be true for $i<m$ and we want to prove that the extension $M_{m}$ is linearly disjoint from $M_{i}$ for every $i<m$. Since the only primes of $k$ which ramify in the extensions $k\left(\sqrt[p]{a_{i}}\right)$ and $k\left(\sqrt[p]{b_{i}}\right)$ are those above $p$ and those dividing the ideals $\left(a_{i}\right)$ and $\left(b_{i}\right)$ respectively, condition (ii) ensures that

$$
k\left(\sqrt[p]{a_{m}}\right) \cap k\left(\sqrt[p]{a_{i}}\right)=k\left(\sqrt[p]{a_{m}}\right) \cap k\left(\sqrt[p]{b_{i}}\right)=k
$$

for every $i<m$. Since $x_{m}$ satisfies the system (4.1), we also have

$$
k\left(\sqrt[p]{a_{m}}\right) \cap k\left(\sqrt[p]{b_{m}}\right)=k .
$$


Moreover, we notice that if $\beta$ is a prime of $k$ dividing $\left(a_{i}\right)$ or $\left(b_{i}\right)$ for $i<m$, then $\beta$ does not divide $\left(b_{m}\right)$; otherwise $\left(x_{m}\right)$ would be divisible by some elements of $S_{m}$. Using the previous arguments we get that $k\left(\sqrt[p]{a_{m}}, \sqrt[p]{b_{m}}\right) \cap k\left(\sqrt[p]{a_{i}}, \sqrt[p]{b_{i}}\right)=k$ for every $i<m$.

We notice that an extraspecial group of order $p^{3}$ has a unique normal subgroup of order $p$, which is given by the center; then the extension $k\left(\sqrt[p]{a_{m}}, \sqrt[p]{b_{m}}\right)$ is the unique $p$-elementary subextension of $M_{m}$ of order $p^{2}$. Therefore the extensions $M_{1}, \ldots, M_{m}$ are linearly disjoint.

We now want to prove condition (b). We denote by $k_{m}$ the compositum of the fields $M_{1}, \ldots, M_{m}$. Then $\operatorname{Gal}\left(k_{m} / k\right)=H_{1} \times \ldots \times H_{m}$ and the field

$$
M=k\left(\sqrt[p]{a_{1}}, \sqrt[p]{b_{1}}, \ldots, \sqrt[p]{a_{m}}, \sqrt[p]{b_{m}}, \sqrt[p]{\omega_{1} \ldots \omega_{m}}\right)
$$

is a subfield of $k_{m}$.

We consider the subgroup $N_{m}$ of $H_{1} \times \ldots \times H_{m}$ described in Proposition 2.1 defined by

$$
N_{m}=\left\{\left(z_{1}^{a_{1}}, \ldots, z_{m}^{a_{m}}\right) \mid a_{1}+\ldots+a_{m} \equiv 0 \bmod p\right\},
$$

where $Z\left(H_{i}\right)=\left\langle z_{i}\right\rangle$. It is easy to see that $z_{i}$ acts in the following way:

$$
\begin{aligned}
& \text { - } z_{i}\left(\sqrt[p]{\omega_{i}}\right)=\zeta_{p} \sqrt[p]{\omega_{i}} \\
& \text { - } z_{i}\left(\sqrt[p]{a_{i}}\right)=\sqrt[p]{a_{i}} \\
& \text { - } z_{i}\left(\sqrt[p]{b_{i}}\right)=\sqrt[p]{b_{i}}
\end{aligned}
$$

The quotient $\left(H_{1} \times \ldots \times H_{m}\right) / N_{m}$ is an extraspecial group of order $p^{2 m+1}$ and exponent $p$ and, by Galois correspondence, the extension $k_{m}^{N_{m}} / k$ is an extraspecial extension of order $p^{2 m+1}$ and exponent $p$. We claim that $M=k_{m}^{N_{m}}$.

The field

$$
k\left(\sqrt[p]{a_{1}}, \sqrt[p]{b_{1}}, \ldots, \sqrt[p]{a_{m}}, \sqrt[p]{b_{m}}\right)
$$

has degree $p^{2 m}$ over $k$ and is contained in $k_{m}^{N_{m}}$, since it is fixed by every element of $N_{m}$. Thus $k_{m}^{N_{m}}$ must be a $p$-extension of this field.

If we take $n=\left(z_{1}^{a_{1}}, \ldots, z_{m}^{a_{m}}\right) \in N_{m}$, we have

$$
n\left(\sqrt[p]{\omega_{1} \cdot \ldots \cdot \omega_{m}}\right)=\zeta_{p}{ }^{a_{1}+\ldots+a_{m}} \sqrt[p]{\omega_{1} \cdot \ldots \cdot \omega_{m}}=\sqrt[p]{\omega_{1} \cdot \ldots \cdot \omega_{m}}
$$

since $\sum_{i=1}^{m} a_{i} \equiv 0 \bmod p$ for $n \in N_{m}$.

Therefore $M \subseteq k_{m}^{N_{m}}$ and, since they have the same degree, they are equal.

4.2. Extension by the group algebra. This section is meant to provide an explicit realization of the field used in the proof of part (iii) of Theorem 2, as announced in Remark 3.5.

To do this we will resort to considerations from Section 2.2 on irreducible modules of extraspecial groups over certain finite fields, the explicit realization of extraspecial groups given in the previous section and a method by Serre.

We start by fixing two odd different primes $p$ and $q$. We consider the family of groups $\left\{E_{m}\right\}_{m}$, where $E_{m}$ is the extraspecial $p$-group of order $p^{2 m+1}$ and exponent $p$ and we denote by $\mathbb{F}_{q}\left[E_{m}\right]$ the group algebra of $E_{m}$ over $\mathbb{F}_{q}$.

We shall now use the method described in 8 , p. 18, to prove that if $F$ is a number field containing the $q$-th root of unity, $L_{m} / F$ is a Galois extension with Galois group $E_{m}$, and $G_{m}$ is the semidirect product $G_{m}=\mathbb{F}_{q}\left[E_{m}\right] \rtimes E_{m}$, then the embedding problem for $L_{m} / F$ and for

$$
1 \rightarrow \mathbb{F}_{q}\left[E_{m}\right] \rightarrow G_{m} \rightarrow E_{m} \rightarrow 1
$$


has a solution. This means that there exists a Galois extension $K_{m}$ of $L_{m}$ such that:

(1) $\operatorname{Gal}\left(K_{m} / L_{m}\right)=\mathbb{F}_{q}\left[E_{m}\right]$;

(2) $K_{m} / F$ is Galois with Galois group isomorphic to $G_{m}$.

In order to construct $K_{m}$, we take a place $v$ of $F$ which splits completely in $L_{m}$ and we consider a place $w$ of $L_{m}$ above $v$. Now we choose an element $x \in L_{m}$ such that $x$ has $w$-adic valuation 1 , but $w_{i}$-adic valuation 0 for every $w_{i} \neq w$ above $v$.

We denote by $\Delta:=\left\{\sqrt[q]{\sigma(x)} \mid \sigma \in E_{m}\right\}$ and we set $K_{m}=L_{m}(\Delta)$. Then the extension $K_{m} / L_{m}$ is a Galois extension with Galois group isomorphic to $\mathbb{F}_{q}\left[E_{m}\right]$. In fact we consider the map

$$
\begin{aligned}
\lambda: \mathbb{F}_{q}\left[E_{m}\right] & \rightarrow \operatorname{Gal}\left(K_{m} / L_{m}\right), \\
\sum_{\sigma \in E_{m}} a_{\sigma} \sigma & \longmapsto \tau,
\end{aligned}
$$

where $\tau$ is defined as $\tau(\sqrt[q]{\sigma(x)})=\zeta_{q}^{a_{\sigma}} \sqrt[q]{\sigma(x)}$; then $\lambda$ is clearly the sought for isomorphism.

The extension $K_{m} / F$ is also a Galois extension: in fact for every $\sigma \in \operatorname{Gal}\left(L_{m} / F\right)$ $=E_{m}$ and every embedding $\tilde{\sigma}: K_{m} \rightarrow F^{\text {alg }}$ with $\left.\tilde{\sigma}\right|_{L_{m}}=\sigma$ we have that, by construction, $\tilde{\sigma}$ is an automorphism of $K_{m}$. Finally, the Galois group $\operatorname{Gal}\left(K_{m} / L_{m}\right)$ is isomorphic to the semidirect product $\mathbb{F}_{q}\left[E_{m}\right] \rtimes E_{m}$, with $E_{m}$ acting on $\mathbb{F}_{q}\left[E_{m}\right]$ by translation.

Now we set $F=\mathbb{Q}\left(\zeta_{p}, \zeta_{q}\right)$ and we take $\left\{L_{m}\right\}_{m}$ to be the family of Galois extensions of $F$ constructed in Section 4.1 with $\operatorname{Gal}\left(L_{m} / F\right)=E_{m}$ the extraspecial group of order $p^{2 m+1}$ and exponent $p$. For every $m$ we use the above method to solve the embedding problem for $L_{m} / F$ and for

$$
1 \rightarrow \mathbb{F}_{q}\left[E_{m}\right] \rightarrow G_{m} \rightarrow E_{m} \rightarrow 1
$$

and we construct a family of number fields $\left\{K_{m}\right\}_{m}$ with Galois groups

$$
\operatorname{Gal}\left(K_{m} / \mathbb{Q}\left(\zeta_{p}, \zeta_{q}\right)\right)=\mathbb{F}_{q}\left[E_{m}\right] \rtimes E_{m} .
$$

In view of Theorem 2 (iii), the compositum $K$ of the family $K_{m}$ is an infinite algebraic extension of $\mathbb{Q}$ with uniformly bounded local degrees which is not contained in $\mathbb{Q}^{(d)}$ for any positive integer $d$.

\section{ACKNOWLEDGMENTS}

The author wishes to thank Professor Umberto Zannier for suggesting the problem and guiding her throughout her Ph.D. thesis. The author is indebted to Professor Andrea Lucchini for providing the construction of the sought family of groups, for useful discussions and for his kind help. She also thanks Professor Roberto Dvornicich for his interest and for useful remarks. Finally, the author is grateful to the unknown referee for the helpful comments which improved the quality of this paper.

\section{REFERENCES}

1. E. Bombieri, U. Zannier, A note on heights in certain infinite extensions of $\mathbb{Q}$. Rend. Mat. Acc. Lincei, 12, 2001, pp. 5-14. MR.1898444 (2003d:11155)

2. S. Checcoli, U. Zannier, On fields of algebraic numbers with bounded local degrees. C. R. Math. Acad. Sci. Paris 349, 2011, pp. 11-14. MR2755687(2012a:11167) 
3. K. Doerk, T. Hawkes, Finite Solvable Groups. De Gruyter, Berlin, 1992. MR1169099 (93k:20033)

4. I. Fesenko, S. Vostokov, Local fields and their extensions, Amer. Math. Soc., Providence, R.I., second edition, 2002. MR.1915966 (2003c:11150)

5. M. D. Fried, M. Jarden, Field Arithmetic, Springer-Verlag, Berlin, 1986. MR868860 (89b:12010)

6. I. Michailov, Four non-abelian groups of order $p^{4}$ as Galois groups. J. Algebra 307, 2007, pp. 287-299. MR2278055 (2008a:12006)

7. W. Narkiewicz, Elementary and Analytic Theory of Algebraic Numbers, Springer-Verlag, Berlin, 1990. MR1055830 (91h:11107)

8. J-P. Serre, H. Darmon, Topics in Galois Theory. Research Notes in Mathematics, Jones and Bartlett Publishers, 1992. MR1162313 (94d:12006)

9. I. R. Shafarevich, On p-extensions. Amer. Math. Soc. Transl., Ser.2, 4, 1956, pp. 59-72.

10. I. R. Shafarevich, Construction of fields of algebraic numbers with given solvable Galois group. Izv. Akad. Nauk SSSR Ser. Mat., 18:6, 1954, pp. 525-578. MR0071469 (17:131d)

11. M. R. Vaughan-Lee, The restricted Burnside problem. Second Ed., Oxford University Press, 1993. MR1364414 (98b:20047)

12. M. Widmer, On certain infinite extensions of the rationals with Northcott property. Monatsh. Math., 2009. MR 2775852

Mathematisches Institut, University of Basel, Rheinsprung 21, CH-4051 Basel, SWITZERLAND 